\title{
Journal of Intelligent Industrial Systems: Preface to Vol. 3 No. 1
}

\author{
G. Rigatos · P. Siano
}

Published online: 24 March 2017

(C) Springer Science+Business Media Singapore 2017

The articles appearing in vol. 3, no. 1 of the Journal of Intelligent Industrial Systems contribute to the development of two research areas: (i) vision-based control and decision making, (ii) nonlinear control of complex dynamical systems. In research area (i), that is vision-based control and decision making, one can distinguish first results about the manufacturing of microphone chips being based on the visual guidance of the microphone's placement on the chip's substrate. This system is capable of providing a high-quality image of the microphone chip, while it can also accomplish high-speed placement requirements by accurately adjusting the chip's center position and rotation angle. A second result in visionbased control is concerned with the real-time recognition of objects transferred by a conveyor belt and their handling by a robot manipulator controlled with the use of PLCs. It is demonstrated that the proposed control scheme assures the fast and accurate accomplishment of pick and place tasks and shows the potential for further development and application at an industrial scale. A third result in vision-based decision making presented in this issue is concerned with the detection of fatigue and drowsiness symptoms in vehicle drivers. The method performs detection of the driver's face and of the eye-pupil position. The pupil positions of the driver are extracted and a ratio is calculated to find the eyes opening. Variations of the latter parameter are used as a criterion to launch a drowsiness alarm. In research area (ii), that is nonlinear control of complex dynamical systems one can distinguish first results on global linearization-based control for the autonomous navigation of robotized underwater vessels. In the proposed approach the 6-DOF dynamic model of the autonomous underwater vessel undergoes a global linearization transformation which allows for the solution of both the control and state estimation problem for this robotic system. A proof is provided about the global asymptotic stability properties of the control method. Finally, another result in the area of advanced nonlinear control is concerned with the generation of electric power out of Proton Exchange Membrane Fuel Cells. A nonlinear optimal control method is developed for this electrochemical system being based on approximate linearization of the dynamic model of the fuel cells around temporary operating points (equilibria). Once again the global asymptotic stability properties of this control method are proven. The content of the articles appearing in the new issue of the journal is outlined as follows.

In article Flying Vision System Design of Placement Machine for Microphone edited by M. Li, J. Cheng, R. Lin, and L. Chen, s microelectromechanical system is developed for the manufacturing of microphone chips. It has been shown that in the die bonding process of microphone chip, the exact center and rotation angle of the microphone chip absorbed by the vacuum nozzle has to be dynamically corrected. In order to achieve a rapid and precise placement, a flying vision system based on a rotating mirror is proposed, The vision system can capture a static clear chip image when a mirror included in it is rotated with reference to its horizontal position. The center position and the rotation angle of chip are fast calculated by a fine and coarse Hough Transform Algorithm. These two parameters are dynamically corrected by the control system while the head of the placement machine approaches to the microphone's targeted position. The experimental results show that the system can obtain high-quality microphone chip image, and that the chips center position and rotating angle correction algorithm can achieve the requirements for accurate positioning of the microphone. In this proposed flying vision system, the mechanical structure is simple and compact, the obtained chips image is free of distortion, and there are no constraints about the mounted head movement trajectory. The flying vision system proposed in the article can significantly enhance the quality and efficiency of microelectomechanical manufacturing processes. 
In article Visual Processing and Classification of items on Moving Conveyor with Pick and Place Robot using PLC edited by S. Kazemi and H. Kharrati, a solution is developed for the problem of PLC-based control of robotic manipulators in pick and place tasks. As noted in the article, many industrial applications require some sort of automated visual processing and classification of items placed on moving conveyors. This paper describes the design and implementation of a robot control system on a hardware platform based on programmable logic controllers (PLCs). The controlled vision based robot is designed for high-performance pick and place applications in packaging work cell. The system includes a pick and place robot whereby a vision system is integrated in its workspace to identify workpieces with respect to their shape and color. A personal computer, operating under the windows platform, carries out all vision related processing and motion planning for the robot. Relevant motion information is communicated through the serial port of the computer to the programmable logic controller (PLC), while the PLC provides also an interface with the sensing and actuation devices of the robot. After sensing and recognizing the objects features, MATLAB sends results to the PLC. In turn, the PLC sends appropriate commands to robots joints, and specifically to the DC and stepper motors comprised in them. The robot picks the object, locates it in its respective destination, and turns back to its reference location. The validity of the developed robot control system is verified through experimental results.

In article $A$ fast and simple drowsiness detection system based on ARM microcontrollers edited by B. MohammadiIvatloo, F. Hasehmazadeh and M. Javad Ostadi, an intelligent system for detection of drowsiness and fatigue symptoms in vehicle drivers is developed. Drowsiness and fatigue of drivers are responsible for severe accidents and may expose to peril both humans and vehicles. This paper presents a method based on image processing for early detection of drivers' drowsiness. To this end, fast and simple algorithms are developed aiming at drivers' face recognition and eyepupil detection. At the first step of the proposed algorithm, the face of the driver is recognized. The eye pupil positions of the driver are extracted and a ratio is calculated indicating the eyes' opening. The latter information is used as a criterion for distinguishing abnormalities related with driver's drowsiness and fatigue symptoms. The proposed method has been first simulated using MATLAB and after getting satisfactory simulation results, it has been implemented in real driving conditions and has been experimentally confirmed. The implemented device can be calibrated easily for each driver in its first use. The proposed drowsiness detection system can be further enhanced and developed with the perspective of use in several types of vehicles.

In article $A U V$ control and navigation with differential flatness theory and Derivative-free nonlinear Kalman Filter- ing edited by G. Rigatos, P. Siano and G. Raffo, the problem of control and navigation for Autonomous Underwater Vessels (AUVs) is solved using differential flatness theory and the Derivative-free nonlinear Kalman Filter. First, differential flatness is proven for the 6-DOF dynamic model of the AUV. This allows for transforming the AUV model into the linear canonical (Brunovsky) form and for designing a state feedback controller. Uncertainty about the parameters of the AUV's dynamic model, as well external perturbations which affect its motion are issues that are taken into account in the controller's design. To compensate for model imprecision and disturbance terms, it is proposed to use a disturbance observer which is based on the Derivative-free nonlinear Kalman Filter. The considered filtering method consists of the standard Kalman Filter recursion applied on the linearized model of the underwater vessel and of an inverse transformation based on differential flatness theory, which enables to obtain estimates of the state variables of the initial nonlinear model of the AUV. With the use of the Kalman Filter-based disturbance observer, simultaneous estimation of the non-measurable state variables of the AUV and of the perturbation terms that affect its dynamics is achieved. Moreover, after estimating such disturbances, their compensation is also succeeded. Simulation experiments are performed to confirm the efficiency of the proposed AUV control and estimation scheme.

Finally, in article $A$ Nonlinear H-Infinity Approach to Optimal Control of PEM Fuel Cells edited by G. Rigatos, P.Siano, P, Wira and J. Liu, a new nonlinear H-infinity control approach is applied to PEM fuel cells. First, the dynamic model of the PEM fuel cells undergoes approximate linearisation, through Taylor series expansion, round local operating points which are defined at each time instant by the present value of the system's state vector and the last value of the control input that was exerted on it. The linearisation procedure requires the computation of Jacobian matrices at the aforementioned operating points. The modelling error, which is due to the truncation of higher order terms in the Taylor series expansion is perceived as a perturbation that should be compensated by the robustness of the control loop. Next, for the linearized equivalent model of the PEM fuel cells, an $\mathrm{H}$-infinity feedback control loop is designed. This approach, is based on the concept of a differential game that takes place between the control input (which tries to minimize the deviation of the state vector from the reference setpoints) and the disturbance input (that tries to maximize it). In such a case, the computation of the optimal control input requires the solution of an algebraic Riccati equation at each iteration of the control algorithm. The known robustness properties of H-infinity control enable compensation of model uncertainty and rejection of the perturbation terms that affect the PEM fuel cells system. The stability of the control loop is proven through Lyapunov analysis. Actually, it is shown that 
H-infinity tracking performance is succeeded, while conditionally the asymptotic stability of the control loop is also assured. The efficiency of the proposed control scheme for the PEM fuel cells model is further confirmed through simulation experiments.

Through the previously analyzed articles, one can perceive the contribution of the new issue of the journal to the dissemination of novel and significant results in the area of intelligent industrial systems. On the one side vision-based control and vision-based decision decision making for industrial systems are fast deploying areas since they can contribute to the precise and rapid accomplishment of several manufacturing tasks. The present issue has given some indicative application examples such as fabrication of microchips and microelectromechanical systems, pick and place tasks in robotic work-cells, and condition monitoring systems for early detection of abnormal conditions in humans or machines. On the other side, complex nonlinear control systems form an extended research field which has to be treated with elaborated control and estimation methods of proven stability and convergence. To this end, the issue has presented application examples about nonlinear control of autonomous underwater vessels being based on global linearization methods. The issue has also provided results on nonlinear control of electric power production from fuel cells, which have made use of approximate linearization methods and optimal control theory. Through the presented results and methods the significance and the impact that the aforementioned two areas have for intelligent industrial systems has been demonstrated and confirmed.

G. Rigatos

Unit of Industrial Automation Industrial Systems Institute 26504 Rion Patras, Greece e-mail: grigat@ieee.org

P. Siano Department of Industrial Engineering University of Salerno 84084 Fisciano, Italy e-mail: psiano@unisa.it 